\title{
A Rotation Scheme for Accurately Computing Meteoroid Flux
}

\author{
Anita Thomas ${ }^{1}$ \\ Advisors: Martin Ratliff ${ }^{2}$ and Shuwang $\mathrm{Li}^{3}$
}

\begin{abstract}
Accurately estimating meteoroid flux helps analyze the risk of collisions while spacecraft move in interplanetary space. Currently, NASA uses the Meteoroid Engineering Model (MEM) to estimate meteoroid flux and the Bumper-ii code to evaluate damage to a spacecraft structure. To help the Bumper algorithm evaluate potential damage, we develop a rotation scheme that converts meteoroid flux information from the reference frame used in the Meteoroid Engineering Model to Bumper's reference frame as a spacecraft rotates in space. Using randomly generated data, we show our average accuracy can be at least $98.6 \%$.
\end{abstract}

Key words. Meteoroid flux, quaternion rotation, solid angle bins.

\section{Introduction}

On February 15, 2013, a meteor explosion in Russia threatened many lives. The meteor fractured 12 to 15 miles above Earth's surface [7]. Broken windows sent shards of glass flying through the air, thus injuring about 1200 people and causing $\$ 30$ million in damages. Because the object was coming from the general direction of the sun, telescopes were unable to detect it in the sun's glare [6]. Videos and news coverage of the distressing event underscore the importance of continued research into meteoroid detection and risk analysis.

While the Russian meteor is a rare example of the threat posed by meteoroids, spacecraft flying through interplanetary space frequently experience more commonplace collisions with particle-sized meteoroids. Here we report on research that helps analyze that meteoroid risk. Currently, NASA uses its Meteoroid Engineering Model (MEM) to estimate meteoroid flux at a given point in space and the NASA Bumper-ii algorithm to evaluate potential damage to a spacecraft structure. Output from MEM is fed into Bumper as input. Because MEM is coded according to a reference frame derived from the spacecraft trajectory and Bumper uses a reference frame fixed to the body of the spacecraft, this process only produces accurate results when the spacecraft maintains a constant orientation $[1,3]$. In this paper we develop an algorithm that rotates meteoroid flux information from MEM's reference frame to Bumper's reference frame as a spacecraft rotates in interplanetary space. This

\footnotetext{
${ }^{1}$ Department of Applied Mathematics, Illinois Institute of Technology, Chicago. thomas1894@gmail.com

${ }^{2}$ Reliability Engineering Office, NASA Jet Propulsion Laboratory, Caltech, J.M.Ratliff@jpl.nasa.gov

${ }^{3}$ Department of Applied Mathematics, Illinois Institute of Technology, Chicago. sli@math.iit.edu
} 
algorithm tracks the change in flux to a given area of the spacecraft, as the spacecraft rotates.

The idea is to visualize a sphere divided into solid-angle bins surrounding a spacecraft. The use of a sphere represents the possibility of a meteoroid collision with a spacecraft from any direction [1]. Each solid-angle bin is assigned its respective meteoroid flux (in pseudo-units of particles per $\mathrm{m}^{2}$ per second per bin solid angle) [3]. When a spacecraft rotates, the bins in MEM's sphere no longer align with the bins in Bumper's sphere. To recalculate the flux in the spacecraft bins, we start by using MEM output to define spacecraft bins in spherical coordinates $(\theta, \phi, r)$. We fill each bin with dots and apportion a fraction of the bin flux to each dot. A rotation then moves the dots to new bins. The fluxes of the new set of dots in each spacecraft bin are summed to determine the new total flux in each bin.

This paper is organized as follows. In section 2, we describe the computing scheme. In section 3, we show examples on accuracy measurement. In section 4, we give concluding remarks and discuss future work.

\section{Description of the Scheme}

\subsection{Quaternion Rotation Matrix}

We use quaternion formalism to determine the dots' new locations after a rotation. An introduction to quaternions can be found in Kuipers [5]. Quaternions rotate coordinate frames in 3-D using rotation information stored in a 4-D vector. The use of quaternions avoids at least some of the singularities and modulus ambiguities that appear in the more commonly used Euler angle representation of a rotation [9].

Quaternions consist of four elements: $q_{1}, q_{2}, q_{3}$ and $q_{4}$. Although they provide no clear visual representation, these four quaternion components together represent a rotation around some axis through some angle of rotation. The quaternion vector that represents a spacecraft rotation about the vector $e$ is defined as

$$
q=\left[\begin{array}{l}
q_{1} \\
q_{2} \\
q_{3} \\
q_{4}
\end{array}\right]=\left[\begin{array}{c}
e_{1} s \\
e_{2} s \\
e_{3} s \\
c
\end{array}\right]
$$

where $e$ is the axis of rotation, $s$ is the value of $\sin \left(\frac{\theta}{2}\right), c$ is the value of $\cos \left(\frac{\theta}{2}\right)$, and $\theta$ is equal to the angle of rotation [9]. The axes of rotation, in this case, correspond to the $x, y$, and $z$ axes. Note that in cases where the axis of rotation is not one of the coordinate axes, find the eigenvector with eigenvalue equal to zero; by definition this eigenvector lies on the vector 
that remains the same before and after rotation (axis of rotation) [9].

Each rotation about the $x, y$, and $z$ axes is represented by its own quaternion vector: $q_{x}, q_{y}$, and $q_{z}$, respectively. These quaternion vectors can be used to find one rotation matrix that represents a collective rotation about the $x, y$, and $z$ axes. To do so, we first calculate the quaternions representing a collective rotation around the $x$ and $y$ axes

$$
q_{x y R o t}=\left[\begin{array}{cccc}
q_{4}^{\prime} & q_{3}^{\prime} & -q_{2}^{\prime} & q_{1}^{\prime} \\
-q_{3}^{\prime} & q_{4}^{\prime} & q_{1}^{\prime} & q_{2}^{\prime} \\
q_{2}^{\prime} & -q_{1}^{\prime} & q_{4}^{\prime} & q_{3}^{\prime} \\
-q_{1}^{\prime} & -q_{2}^{\prime} & -q_{3}^{\prime} & q_{4}^{\prime}
\end{array}\right] q_{x}
$$

where $q_{x}$ is the quaternion rotation vector representing a rotation around the $x$-axis and $q_{1}^{\prime}, q_{2}^{\prime}, q_{3}^{\prime}, q_{4}^{\prime}$ represent the quaternion components of $q_{y}[9]$.

We then calculate the quaternions corresponding to a collective rotation around the $x, y$, and $z$ axes, respectively,

$$
q_{x y z R o t}=\left[\begin{array}{cccc}
q_{4}^{\prime \prime} & q_{3}^{\prime \prime} & -q_{2}^{\prime \prime} & q_{1}^{\prime \prime} \\
-q_{3}^{\prime \prime} & q_{4}^{\prime \prime} & q_{1}^{\prime \prime} & q_{2}^{\prime \prime} \\
q_{2}^{\prime \prime} & -q_{1}^{\prime \prime} & q_{4}^{\prime \prime} & q_{3}^{\prime \prime} \\
-q_{1}^{\prime \prime} & -q_{2}^{\prime \prime} & -q_{3}^{\prime \prime} & q_{4}^{\prime \prime}
\end{array}\right] q_{x y R o t}
$$

where $q_{1}^{\prime \prime}, q_{2}^{\prime \prime}, q_{3}^{\prime \prime}, q_{4}^{\prime \prime}$ represent the quaternion components of $q_{z}[9]$.

When used in this direction cosine matrix

$$
A=\left[\begin{array}{ccc}
q_{1}^{2}-q_{2}^{2}-q_{3}^{2}-q_{4}^{2} & 2\left(q_{1} q_{2}+q_{3} q_{4}\right) & 2\left(q_{1} q_{3}-q_{2} q_{4}\right) \\
2\left(q_{1} q_{2}-q_{3} q_{4}\right) & -q_{1}^{2}+q_{2}^{2}-q_{3}^{2}+q_{4}^{2} & 2\left(q_{2} q_{3}+q_{1} q_{4}\right) \\
2\left(q_{1} q_{3}+q_{2} q_{4}\right) & 2\left(q_{2} q_{3}-q_{1} q_{4}\right) & -q_{1}^{2}-q_{2}^{2}+q_{3}^{2}+q_{4}^{2}
\end{array}\right]
$$

the quaternion components of $q_{x y z \text { Rot }}$ form a $3 \times 3$ rotation matrix equal to its corresponding Euler rotation matrix [9]. 


\subsection{Defining Spacecraft Bins}

In this section, we provide an explanation of MEM's outputs and discuss how that output is reformatted for use in our rotation scheme. Figure 2.1 shows the top half of the solid angle bin pattern corresponding to MEM output [1].

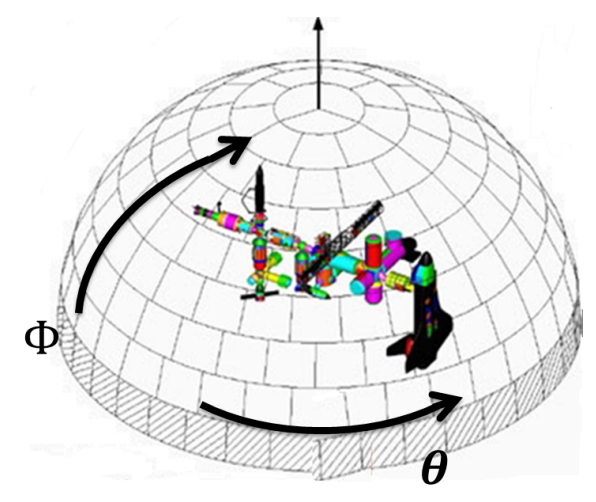

Figure 2.1: MEM output: solid angle bin pattern

In Figure 2.1, the angle $\phi$ refers to the elevation of a spacecraft bin and ranges from $-\frac{\pi}{2}$ to $\frac{\pi}{2}$. The angle $\theta$ shows how far around the sphere a bin is located and ranges from zero to $2 \pi[1]$.

Table 2.1 is a subset of MEM output.

\begin{tabular}{|c|c|c|c|c|c|c|}
\hline $\mathrm{A}$ & $\mathrm{B}$ & $\mathrm{C}$ & $\mathrm{D}$ & $\mathrm{E}$ & $\mathrm{F}$ & $\mathrm{G}$ \\
\hline 1 & 1 & 1 & -90 & -85 & 0 & 120 \\
\hline 2 & 1 & 2 & -90 & -85 & 120 & 240 \\
\hline 3 & 1 & 3 & -90 & -85 & 240 & 360 \\
\hline 4 & 2 & 1 & -85 & -80 & 0 & 40 \\
\hline 5 & 2 & 2 & -85 & -80 & 40 & 80 \\
\hline 6 & 2 & 3 & -85 & -80 & 80 & 120 \\
\hline 7 & 2 & 4 & -85 & -80 & 120 & 160 \\
\hline 8 & 2 & 5 & -85 & -80 & 160 & 200 \\
\hline 9 & 2 & 6 & -85 & -80 & 200 & 240 \\
\hline 10 & 2 & 7 & -85 & -80 & 240 & 280 \\
\hline 11 & 2 & 8 & -85 & -80 & 280 & 320 \\
\hline 12 & 2 & 9 & -85 & -80 & 320 & 360 \\
\hline
\end{tabular}

Table 2.1: MEM output (subset) 
Column A defines a numbering scheme for the bins. Column B dentoes the row that a bin lies in, and the column $\mathrm{C}$ indicates a bin's location within its respective row. Columns $\mathrm{D}$, E, F, G of the MEM output represent the start and end $\phi$ and $\theta$ of every spacecraft bin. In order to define the MEM angles in terms of standard spherical coordinates, the algorithm employs the following procedure:

1. Change all angles from degrees to radians.

2. Switch the columns MEM calls $\theta$ and $\phi$ so that $\theta$ represents elevation and $\phi$ represents the azimuth angle.

3. Flip the $\theta$ start and $\theta$ end vectors top to bottom to define bin one at the top of the sphere.

4. Interchange the $\theta$ start and $\theta$ end vectors.

5. Subtract all angles in the $\theta$ start and $\theta$ end vectors from $\frac{\pi}{2}$, so that $\theta$ ranges from 0 to $\pi$.

As an example, Figure 2.2 shows an arbitrary bin after the above modifications have been implemented and Table 2.2 shows the $\theta$ and $\phi$ bounds of the twelve bins at the "top" of the sphere after the above modifications have been implemented.

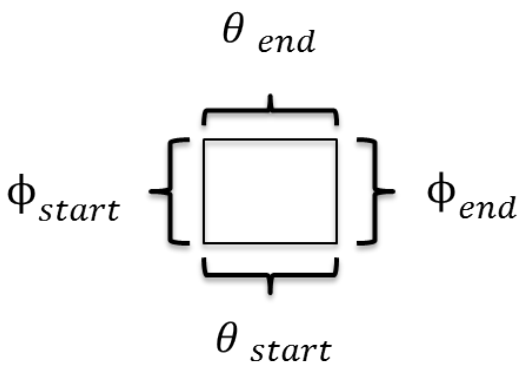

Figure 2.2: MEM output: solid angle bin example 


\begin{tabular}{|c|c|c|c|}
\hline$\theta_{\text {start }}$ & $\theta_{\text {end }}$ & $\phi_{\text {start }}$ & $\phi_{\text {end }}$ \\
\hline 0 & 0.0873 & 0 & 2.0944 \\
\hline 0 & 0.0873 & 2.0944 & 4.1888 \\
\hline 0 & 0.0873 & 4.1888 & 6.2832 \\
\hline 0.0873 & 0.1745 & 0 & 0.6981 \\
\hline 0.0873 & 0.1745 & 0.6981 & 1.3963 \\
\hline 0.0873 & 0.1745 & 1.3963 & 2.0944 \\
\hline 0.0873 & 0.1745 & 2.0944 & 2.7925 \\
\hline 0.0873 & 0.1745 & 2.7925 & 3.4907 \\
\hline 0.0873 & 0.1745 & 3.4907 & 4.1888 \\
\hline 0.0873 & 0.1745 & 4.1888 & 4.8869 \\
\hline 0.0873 & 0.1745 & 4.8869 & 5.5851 \\
\hline 0.0873 & 0.1745 & 5.5851 & 6.2832 \\
\hline
\end{tabular}

Table 2.2: MEM output modification: subset of bin $\theta$ and $\phi$ bounds

\subsection{Defining Dots on Sphere}

In this section, we explain one successful and three less successful means of defining dots on the sphere. The successful method is described below and is immediately followed by descriptions of the three less successful attempts.

When a spacecraft rotates, the bins on the MEM and Bumper spheres become unaligned. To recalculate the flux in the spacecraft bins after rotation, the fraction of each MEM bin that rotates into a spacecraft bin is determined and the corresponding flux in each of those fractions is summed to define the new total flux in that spacecraft bin. In order to find the fraction of each MEM bin that rotates into a spacecraft bin, each bin is filled with dots and the fraction of dots that rotate into different spacecraft bins reveal the fraction of each MEM bin that rotates into a spacecraft bin.

Each bin has an associated meteoroid flux (in pseudo-units of particles per $\mathrm{m}^{2}$ per second per bin solid angle) [3]. Each dot in a bin represents a fraction of that flux. Since each dot in a bin is assigned the same fraction of total flux per bin, it is important to distribute the dots so that they also represent the same fraction of surface area within a bin. Because the surface area of a bin varies with $\theta$, more dots are assigned to areas of a bin with $\theta$ values closer to the equator. To accomplish this skewed distribution, the algorithm divides each bin into ten levels, as shown in Figure 2.3. 

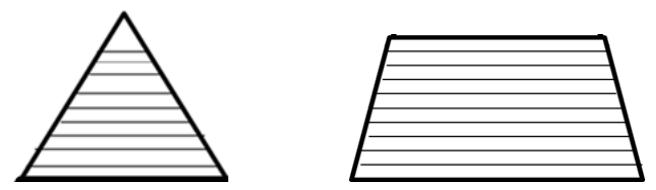

Figure 2.3: Bin shapes

The triangle represents the bins at the poles and the trapezoid represents all other bins. Note that bins on the bottom of the sphere are inverted. We will refer to the total number of dots per bin and the total surface area per bin as $N$ and $S$, respectively. We will refer to the total number of dots per level and the total surface area per level as $n$ and $s$, respectively. The surface area in steradians of each level, $s$, within each bin is calculated and then multiplied by the number of dots per steradian-calculated by dividing a user-defined value for the number of dots per bin, $N$, by the surface area of each bin, $S$. The resulting number, rounded to the nearest whole number, represents the number of randomly generated dots per level, $n$, in each bin, or more compactly

$$
n=\frac{(s)(N)}{S}
$$

This approach creates a fairly constant spatial distribution of dots within a bin, which results in an even distribution of flux across the bin when each dot is assigned the same flux per dot within the bin. Flux per dot is determined by dividing the flux per bin by the number of dots in the bin.

Before implementing the distribution described above, we tested three other distributions. The corresponding accuracies are summarized and explained in section 3 of this paper. These distributions include

\section{- Constant Step Size}

Using a constant step size, we defined each dots' $\theta$ coordinate from 0 to $\pi$ and $\phi$ coordinate from 0 to $2 \pi$. Each row of dots has an equal number of dots and each column has an equal number of dots.

- Uniform Distribution

The agorithm generates uniformly distributed random numbers between 0 and $\pi$ for $\theta$ and between 0 and $2 \pi$ for $\phi$.

We attributed the less than acceptable accuracy of the above two distributions to the increasing surface area of a sphere as $\theta$ nears the equator. As a result, we sought to implement a distribution that accounts for this change in surface area by placing an increasing number of dots in the bins as they neared the equator; this idea led to a Chebyshev distribution. 


\section{- Chebyshev Nodes}

Chebyshev polynomials of the first kind contain roots, or nodes, that interpolate polynomials in the interval $[-1,1]$. In this case, -1 represents the bottom of the sphere and 1 represents the top of the sphere. Chebyshev distributes points more heavily at the poles, which is the opposite of what we needed. To adjust, we created two intervals, shifted them $\frac{\pi}{2}$ up and down, and used half of each interval [4]. We refer to the total number of dots on the sphere as $M$.

The formulas we used to determine the $\theta$ part of the coordinates are

$$
\begin{aligned}
& x_{k}=\cos \left(\frac{2 k+1}{2 n+2} \pi\right), \\
& \theta_{k}=\frac{\pi}{2}\left(x_{k}+1\right) \pm \frac{\pi}{2},
\end{aligned}
$$

where $k=1,2, . ., M[4]$.

To determine the corresponding $\phi$ part of the coordinates, we subtracted $\theta_{k}-\theta_{k-1}$ and assigned that value as the step size for $\phi$. So, $\phi_{k}$ ranges from 0 to $2 \pi$ with stepsize $\theta_{k}-\theta_{k-1}$.

In total, we tested four distributions. The first three distributions refer to the first three we coded-the less successful distributions. The fourth distribution refers to the final, successful distribution.

\subsection{Rotating Dots}

In this section, we present the formulas used to rotate the dots on the sphere. To rotate the dots through any angle about any axis, the dots' coordinates are first changed from spherical to Cartesian and then multiplied by a rotation matrix. The rotated Cartesian coordinates are then transferred back into spherical coordinates.

The formulas

$$
\begin{gathered}
x=r \sin (\theta) \cos (\phi), \\
y=r \sin (\theta) \sin (\phi), \\
z=r \cos (\theta),
\end{gathered}
$$

change the dots from spherical to Cartesian points where $r=1$ on a unit sphere [8]. 
The formula

$$
q R=\left[\begin{array}{ccc}
q_{1}^{2}-q_{2}^{2}-q_{3}^{2}-q_{4}^{2} & 2\left(q_{1} q_{2}+q_{3} q_{4}\right) & 2\left(q_{1} q_{3}-q_{2} q_{4}\right) \\
2\left(q_{1} q_{2}-q_{3} q_{4}\right) & -q_{1}^{2}+q_{2}^{2}-q_{3}^{2}+q_{4}^{2} & 2\left(q_{2} q_{3}+q_{1} q_{4}\right) \\
2\left(q_{1} q_{3}+q_{2} q_{4}\right) & 2\left(q_{2} q_{3}-q_{1} q_{4}\right) & -q_{1}^{2}-q_{2}^{2}+q_{3}^{2}+q_{4}^{2}
\end{array}\right]\left[\begin{array}{c}
x_{i} \\
y_{i} \\
z_{i}
\end{array}\right]
$$

where $q_{1}, q_{2}, q_{3}$, and $q_{4}$ are the quanternion components of $q_{x y z R o t}$ and $x_{i}, y_{i}$, and $z_{i}$ are the Cartesian components of each dot, rotates all the dots [9].

To convert the rotated dots from Cartesian to spherical, use the following formulas

$$
\begin{aligned}
\theta & =\cos ^{-1}\left(\frac{z}{r}\right), \\
\phi & =\tan ^{-1}\left(\frac{y}{x}\right),
\end{aligned}
$$

with $r=1$ [8].

Because the range of arctan spans $\frac{\pi}{2}$ to $-\frac{\pi}{2}$, the algorithm checks the $x$ and $y$ components of each rotated dot to determine which quadrant $\phi$ was supposed to end up in. If $x$ is negative and $y$ is positive, $\pi$ is added to $\phi$. If $x$ is negative and $y$ is negative, $\pi$ is also added to $\phi$. Finally, if $x$ is positive and $y$ is negative, $2 \pi$ is added to $\phi$.

\subsection{Calculating New Flux}

To identify the bin each dot rotates into, the spherical coordinates of each dot are compared to the start and end spherical coordinates of each bin. The fluxes of the dots in each bin are summed to determine the new flux in each spacecraft bin. The flux is then flipped from top to bottom to adhere to the bin numbering system used by Bumper. The overall algorithm is in Algorithm 1. 


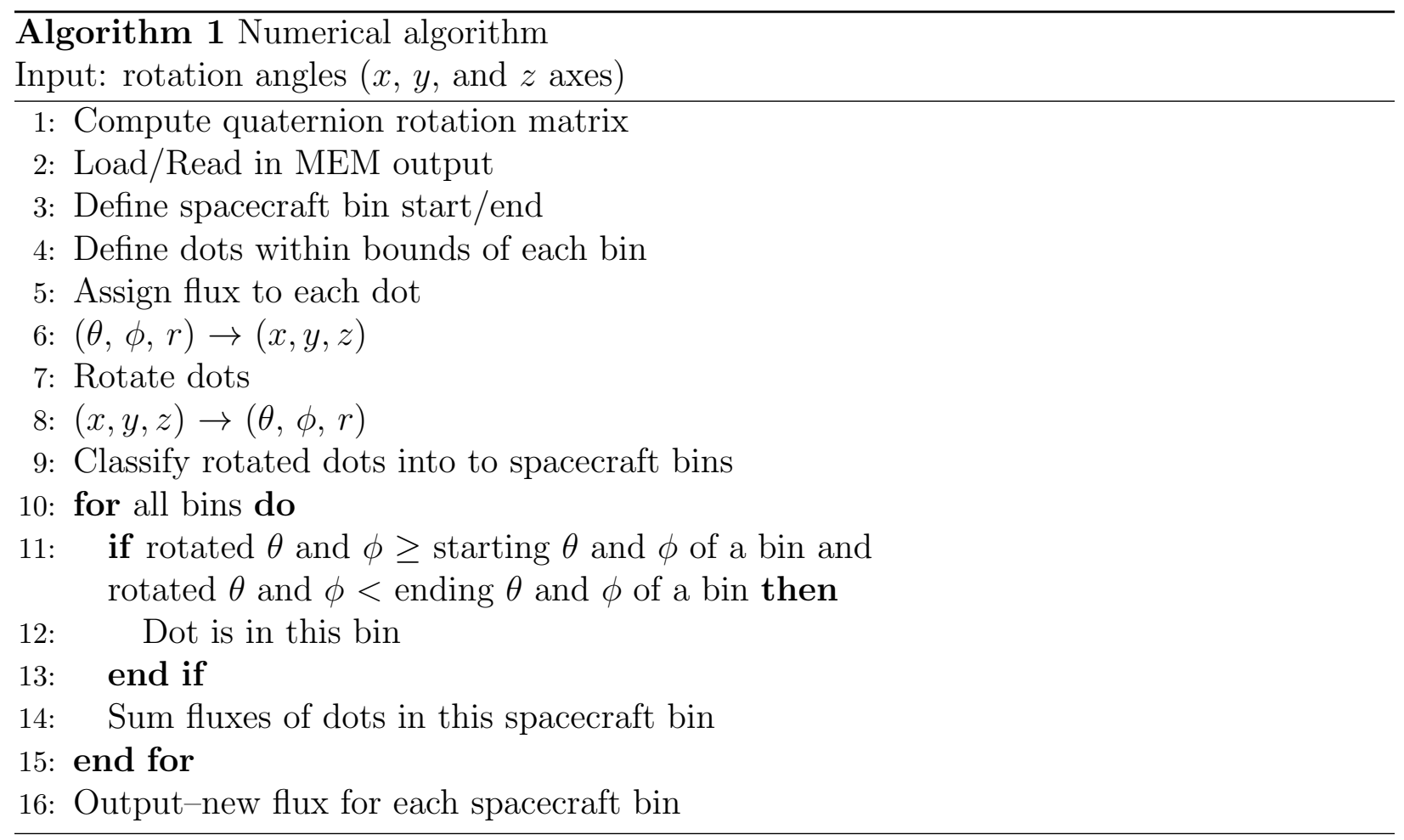

\section{Results of Accuracy Measurement}

As mentioned, we tested three distributions prior to finding one that produced desirable accuracy. To determine accuracy, we needed to compare the algorithm's answers for postrotation flux per bin with the true post-rotation flux per bin. Because the surface area of each bin remains constant before and after rotation, we were able to use the surface area of each bin as simulated flux data. The percent accuracy of the flux, or in this case surface area, in each bin is computed by comparing surface area before and after rotation. We deonote the percent accuracy with $A$ and compute the percentage using

$$
A=100-100\left(\frac{S-S^{\prime}}{S}\right),
$$

where $S$ is the surface area of a bin before rotation and $S^{\prime}$ is the surface area of a bin after rotation [2].

To test the accuracy of each distribution, we ran a series of trials consisting of randomly generated $x, y$, and $z$ angles between 0 and $2 \pi$. The surface area of each bin was assigned as flux per bin. We computed the average accuracy of the flux in each bin over all the trials, as well as the maximum percent error shown in at least one bin in each of the trials. The first three distributions we tried, assigned about 800,000 dots total on a sphere. Results showed several spikes in the average accuracy of each bin. For example, 25 bins in a row may have 
an average accuracy between $93-99 \%$ and then the next bin may drop down to $62 \%$ average accuracy. The maximum percent error per trial for these three distributions ranged from about $15 \%$ to $62 \%$ for the constant step size distribution, about $37.3 \%$ to $38.8 \%$ for the uniform distribution, and about $125.6 \%$ for the Chebyshev distribution.

We found that the source of the spikes in average accuracy involved the uneven distributon of flux throughout a bin-especially in the bins near the poles. This discovery led to the fourth and final dot distribution explained in the beginning of section 2.3 of this paper. To test this distribution, we ran 50 trials of randomly generated $x, y$, and $z$ angles between 0 and $2 \pi$. Each bin was assigned 2500 dots. The following graphs show the average accuracy of each bin over 50 trials with the bin number on the $x$ axis and the average percent accuracy on the $y$ axis. While MEM divides a sphere into 1652 bins, the plots show only a quarter of the bins at a time for easier viewing. Every bin operates at an average of at least $98.6 \%$ accuracy.

The plots in Figure 3.1 illustrate average accuracy. The lowest average accuracy of the fourth distribution $(\approx 98.6 \%)$ is significantly improved from the spikes in average accuracy of the first three distributions, which dropped as low as about $91 \%$ in the constant step size distribution, about $62 \%$ in the uniform distribution, and about $-25 \%$ in the Chebyshev distribution. 

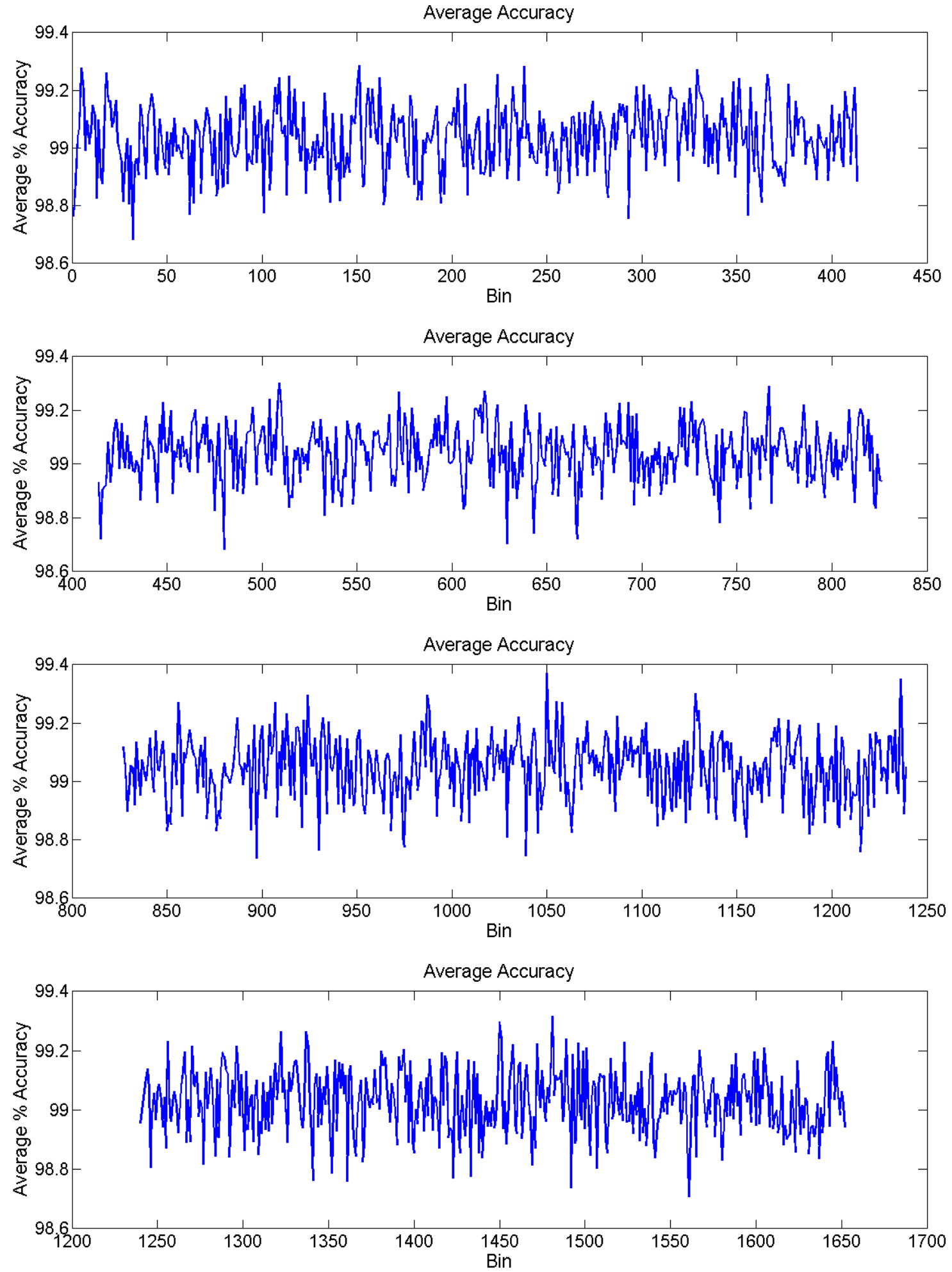

Figure 3.1: Results: Average Accuracy 
Figure 3.2 shows the maximum percent error shown in at least one bin in each of the 50 trials. At any time, any bin can show as little as about $3.7 \%$ error or as much as about $5.7 \%$ error.

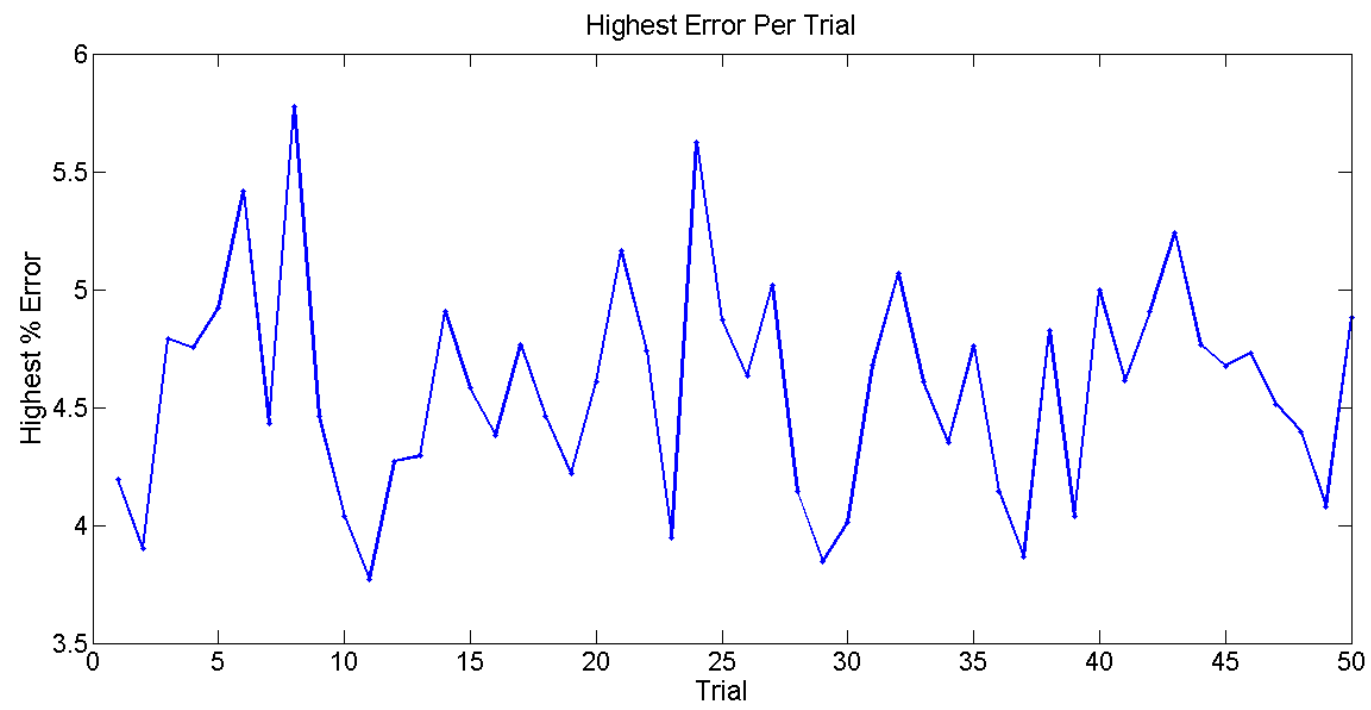

Figure 3.2: maximum percent error

The highest error per trial using the fourth distribution $(\approx 5.7 \%)$ is significantly improved from the highest errors per trial associated with the first three distributions, which were as high as about $62 \%$ in the constant step size distribution, about $38.8 \%$ in the uniform distribution, and about $125.6 \%$ in the Chebyshev distribution.

\section{Conclusion}

The improvement in accuracy from the first three dot distributions to the fourth dot distribution is significant. This increase is largely due to alleviating the overcrowding at the poles of the sphere where the bins are shaped like triangles. Of all the bins on the sphere, the surface area increases most rapidly in the first three bins as $\theta$ increases, and the surface area decreases most rapidly in the last three bins as $\theta$ decreases. The dots closest to the poles were assigned flux values much too large for the actual flux they represented, and therefore, misconstrued the new flux in the bins they rotated to. Without the adjustments made in the fourth distribution, tightly clustered dots were assigned the same flux as the more loosely clustered dots, which led to errors in the post-rotation flux even though the dots were rotating correctly and were classified into bins correctly.

Given the accuracy of the fourth distribution's results, this algorithm is an acceptable rotation scheme for computing meteoroid flux and could be implemented as a direct feed from 
MEM to Bumper. For spacecraft that vary their orientation as they travel through space, implementation of this flux rotation algorithm will provide a simpler, more accurate means of determining the threat of meteoroid impact.

\section{Future Work}

- Implement this algorithm as a direct feed from MEM to Bumper, adjusting inputs and outputs as needed.

- Make the algorithm more time efficient if necessary (current runtime: about 24 minutes).

- Adjust accuracy as needed by increasing/decreasing number of dots per bin.

\section{Acknowledgements}

I began this research as a summer intern at NASA Jet Propulsion Laboratory/Caltech under the guidance of Martin Ratliff, who has earned my first thank you. He took time out of each day to help guide me in the right direction. He genuinely cared that I had a fun and educational experience in California. I'd also like to thank Martin for submitting recommendation letters that helped me obtain another summer internship with NASA and a fellowship to OSU's Geodetic Science Masters Program. I'd like to thank Dr. Shuwang Li for agreeing to advise me throughout the Spring 2013 semester. He's proposed many great ideas, taught me to always remain positive and smile, and also helped me obtain a summer internship and fellowship for my graduate studies.

From my time at JPL, I'd like to thank Dr. Randall Swimm for acting as my unofficial mentor and showing patience when explaining the math I needed to know for my project. Thank you to John Campbell who always took an interest in my future and offered me helpful advice. Thank you to Eliza O'Reilly for offering helpful suggestions as I completed this research and thank you to Katelyn Kufahl for livening up my office experience. I'd like to thank everyone who works in JPL's building 122 for making my experience more valuable than I could have imagined. Thank you to all the people working to make NASA's MUST (Motivating Undergraduates in Science and Technology) and MSP (Minority Students Program) summer programs successful. Finally, thank you to our reviewers for their helpful comments.

\section{References}

[1] Christiansen, Eric L. Meteoroid/Debris Shielding. http://ntrs.nasa.gov/search. jsp?R=20030068423, NASA, Aug. 2003. Accessed on June 7, 2012. 
[2] Imaging the Universe. http://astro.physics.uiowa.edu/ITU/glossary/ percent-error-formula/, University of Iowa, 2012. Accessed on August 3, 2012 .

[3] Jones, J. Meteoroid Engineering Model-Final Report. http://see.msfc.nasa.gov/ mod/SEECR-2004-400_MOD_MEM. pdf, NASA Marshall Space Flight Center, June 2004. Accessed on June 26, 2014.

[4] Kincaid, David and Ward Cheney, Numerical Analysis: Mathematics of Scientific Computing. Brooks/Cole, Pacific Grove, California, 3rd edition, 2002.

[5] Kuipers, Jack, Quaternions and Rotation Sequences. Princeton University Press, Princeton, New Jersey, 1999.

[6] Kuzmin, Andrey. Meteorite explodes over Russia, more than 1,000 injured. www.reuters.com/article/2013/02/15/ us-russia-meteorite-idUSBRE91E05Z20130215, Reuters, Feb. 2013. Accessed on May 1, 2013.

[7] Phillips, Tony. What Exploded over Russia?. science.nasa.gov/science-news/ science-at-nasa/2013/26feb_russianmeteor/, NASA, Feb. 2013. Accessed on May $1,2013$.

[8] Review B: Coordinate Systems. web.mit.edu/8.02t/www/materials/modules/ ReviewB.pdf, MIT. Accessed on June 15, 2012.

[9] Edited by James Wertz. Spacecraft Attitude Determination and Control, D. Reidel Publishing Company, Dordrecht, Holland, 1978. 